\title{
Effects of nintedanib in patients with idiopathic pulmonary fibrosis by GAP stage
}

\author{
Christopher J. Ryerson ${ }^{1}$, Martin Kolb (i) ${ }^{2}$, Luca Richeldi ${ }^{3}$, Joyce Lee ${ }^{4}$, \\ Daniel Wachtlin ${ }^{5}$, Susanne Stowasser ${ }^{5}$ and Venerino Poletti ${ }^{6}$
}

Affiliations: ${ }^{1}$ Dept of Medicine and Centre for Heart Lung Innovation, University of British Columbia, Vancouver, BC, Canada. ${ }^{2}$ Dept of Medicine, McMaster University, Hamilton, ON, Canada. ${ }^{3}$ Fondazione Policlinico A. Gemelli IRCCS, Università Cattolica del Sacro Cuore, Rome, Italy. ${ }^{4}$ Dept of Medicine, University of Colorado Denver, Aurora, CO, USA. ${ }^{5}$ Boehringer Ingelheim International GmbH, Ingelheim am Rhein, Germany. ${ }^{6}$ Dept of Medicine, Aarhus University Hospital, Aarhus, Denmark.

Correspondence: Christopher J. Ryerson, St Paul's Hospital, 1081 Burrard St, Ward 8B, Vancouver, BC, V6Z 1Y6, Canada. E-mail: chris.ryersonahli.ubc.ca

ABSTRACT We conducted a post hoc analysis to assess the potential impact of GAP (gender, age, physiology) stage on the treatment effect of nintedanib in patients with idiopathic pulmonary fibrosis.

Outcomes were compared in patients at GAP stage I versus II/III at baseline in the INPULSIS ${ }^{\circ}$ trials.

At baseline, 500 patients were at GAP stage I (nintedanib 304, placebo 196), 489 were at GAP stage II (nintedanib 296, placebo 193) and 71 were at GAP stage III (nintedanib 38, placebo 33). In nintedanibtreated patients, the annual rate of decline in forced vital capacity (FVC) was similar in patients at GAP stage I and GAP stage II/III at baseline $\left(-110.1\right.$ and $-116.6 \mathrm{~mL} \cdot$ year $^{-1}$, respectively), and in both subgroups was lower than in placebo-treated patients $\left(-218.5\right.$ and $-227.6 \mathrm{~mL} \cdot y e a r^{-1}$, respectively) (treatment-by-time-by-subgroup interaction $\mathrm{p}=0.92$ ). In the nintedanib group, the number of deaths was $43.8 \%$ of those predicted based on GAP stage (35 versus 79.9). In the placebo group, the number of deaths was $59.8 \%$ of those predicted based on GAP stage ( 33 versus 55.2 ).

In conclusion, data from the INPULSIS ${ }^{\circ}$ trials suggest that nintedanib has a similar beneficial effect on the rate of FVC decline in patients at GAP stage I versus II/III at baseline.

@ERSpublications

Nintedanib provides a similar benefit versus placebo on the rate of decline in forced vital capacity in patients with idiopathic pulmonary fibrosis irrespective of GAP stage at baseline http://ow.ly/HfJ730nNkRT

Cite this article as: Ryerson $\mathrm{CJ}$, Kolb M, Richeldi L, et al. Effects of nintedanib in patients with idiopathic pulmonary fibrosis by GAP stage. ERJ Open Res 2019; 5: 00127-2018 [https://doi.org/ 10.1183/23120541.00127-2018].

This article has supplementary material available from openres.ersjournals.com.

The INPULSIS trials are registered at www.clinicaltrials.gov with identifier numbers NCT01335464 and NCT01335477. Data are available upon request. A request can be submitted at https://trials.boehringer-ingelheim.com/trial_results/ clinical_submission_documents.html.

Received: Aug 032018 | Accepted after revision: Feb 082019

Copyright $\odot$ ERS 2019. This article is open access and distributed under the terms of the Creative Commons Attribution Non-Commercial Licence 4.0. 


\section{Introduction}

Idiopathic pulmonary fibrosis (IPF) is a chronic and progressive interstitial lung disease (ILD) that is characterised by loss of lung function, reduced quality of life, acute exacerbations and high mortality [1]. The median survival of patients with IPF is $\sim 3$ years from the time of diagnosis [2].

Baseline and changes from baseline in individual physiological variables, including forced vital capacity (FVC) and diffusing capacity of the lungs for carbon monoxide (DLCO), have been shown to be predictors of mortality in patients with IPF [3-6]. Several composite risk prediction scores have been developed with the aim of more accurately predicting mortality. The GAP (gender, age, physiology) index and staging system was designed to be a quick and simple method for estimating mortality risk [7]. The GAP index is based on four commonly measured variables (sex, age, FVC \% predicted, DLCO \% pred) and was validated in three cohorts of patients in a real-world setting [7].

Nintedanib is a tyrosine kinase inhibitor that slows disease progression by reducing the rate of decline in lung function in patients with IPF $[8,9]$. Nintedanib has an acceptable safety profile and is approved for the treatment of IPF in many countries and regions, including the USA, Canada, Europe and Japan. Nintedanib has consistent effects in multiple subgroups of patients with IPF [10-14]; however, it is unknown whether the impact of nintedanib is similar across GAP stages, or whether a staging system could facilitate future clinical trials by enriching study cohorts for patients at greater risk for outcomes of interest. The objective of this study was to assess the potential impact of GAP stage on the benefits, tolerability and safety of nintedanib in patients with IPF.

\section{Methods}

\section{Study design}

The INPULSIS $^{\circledR}$ trials were two replicate, randomised, double-blind, phase III trials that compared the effects of nintedanib $150 \mathrm{mg}$ twice daily with placebo over 52 weeks of treatment in patients with IPF [8]. The studies included 1066 patients from 205 sites in 24 countries, of whom 1061 received at least one dose of study drug, with a 3:2 randomisation of nintedanib to placebo. To be eligible for these trials, patients had to be aged $\geqslant 40$ years, have an FVC $\geqslant 50 \%$ pred and have a DLCO $30-79 \%$ pred. The primary end-point in both trials was the annual rate of decline in FVC $\left(\mathrm{mL} \cdot y e \mathrm{rr}^{-1}\right)$. Key secondary end-points were the time to first investigator-reported acute exacerbation over 52 weeks and change from baseline in St George's Respiratory Questionnaire (SGRQ) total score, a measure of health-related quality of life, over 52 weeks. Details of the methodology used in the INPULSIS ${ }^{\oplus}$ trials have been published $[8,15]$.

\section{Study measurements}

FVC was measured using standardised equipment according to established recommendations [16] at baseline, at weeks 2, 4, 6, 12, 24, 36 and 52, and at a follow-up visit 4 weeks later. Patients who prematurely discontinued trial medication were asked to complete all visits as planned. DLCO (corrected for haemoglobin) was measured at baseline and at weeks 24 and 52 using the investigator's own equipment and in compliance with American Thoracic Society/European Respiratory Society guidelines on single-breath determination of carbon monoxide uptake [17].

Acute exacerbations of IPF were defined as events meeting all of the following criteria: unexplained worsening or development of dyspnoea within 30 days; new diffuse pulmonary infiltrates on chest radiography and/or high-resolution computed tomography or the development of parenchymal abnormalities with no pneumothorax or pleural effusion (new ground glass opacities) since the preceding visit; and exclusion of any known causes of acute worsening in accordance with routine clinical practice and microbiological studies. Acute exacerbations that occurred up to 52 weeks plus a 1-week margin after randomisation were included in these analyses.

The SGRQ was measured at baseline and at weeks 6, 12, 24 and 52. The SGRQ is a patient-reported respiratory disease-specific quality-of-life questionnaire that includes three domains (symptoms, activity, impact) [18]. Scores for each domain and the total score are weighted so that they range from 0 to 100 , with higher scores indicating worse health-related quality of life. The minimally important difference in patients with IPF has been estimated to be between 4 and 10 points $[19,20]$.

Safety was measured by clinical and laboratory evaluation and the recording of adverse events from the first dose of study drug until 28 days after the last dose of study drug.

\section{GAP index and stage}

In the GAP index and staging system, points are assigned to each variable (sex, age, FVC, DLCO) to obtain a total point score (GAP index) ranging from 0 to 8 [7]. The GAP index is used to classify patients as at GAP stage I ( $0-3$ points), GAP stage II ( $4-5$ points) or GAP stage III (6-8 points). Based on data from 
228 patients in a derivation cohort (followed for a median 1.7 years) and 330 patients in a validation cohort (followed for a median 2.4 years), model-predicted estimates of mortality at 1 year were $5.6 \%$, $16.2 \%$ and $39.2 \%$ for patients at GAP stages I, II and III, respectively [7]. In this analysis, the GAP index and stage were calculated for all patients in whom the relevant baseline assessments were available.

\section{Statistical analysis}

Post hoc subgroup analyses were conducted using pooled data from the two INPULSIS ${ }^{\bullet}$ trials. Patients were stratified by baseline GAP stage. Analyses were conducted on subgroups of patients by individual GAP stage (I, II, III) and by GAP stage I versus II/III, given the small number of patients at GAP stage III. The primary end-point (annual rate of decline in FVC) and key secondary end-points (time to first investigator-reported acute exacerbation and change from baseline in SGRQ total score) were analysed using the original statistical approach within each GAP stage subgroup. For the primary end-point, the subgroup and the interaction terms treatment-by-subgroup, time-by-subgroup and treatment-by-time-bysubgroup were included in the model. For the key secondary end-points, the subgroup and a treatmentby-subgroup interaction term were included in the model. Time to disease progression (defined as absolute FVC decline $\geqslant 5 \%$ pred or death, or absolute FVC decline $\geqslant 10 \%$ pred or death) over 52 weeks was assessed using a Cox's regression model with the subgroup and an interaction term treatment-bysubgroup included. For all the subgroup analyses, the presence of a statistically significant interaction term could indicate a difference in the impact of nintedanib versus placebo across GAP stages. Safety analyses were descriptive.

\section{Results}

\section{Patients}

At baseline, GAP index and stage could be calculated in 1060 patients treated with at least one dose of study drug (supplementary table S1). Data on baseline DLCO were not available for one patient treated with placebo. The study population comprised 500 patients at GAP stage I (nintedanib 304, placebo 196), 489 at GAP stage II (nintedanib 296, placebo 193), and 71 at GAP stage III (nintedanib 38, placebo 33). Baseline characteristics were generally balanced between the nintedanib and placebo arms within the GAP stage I and GAP stage II/III subgroups (table 1). Patients at GAP stage II/III were older, more likely to be male and had lower FVC and DLCO \% pred compared with patients at GAP stage I. Baseline FVC and DLCO \% pred for each GAP index and stage are summarised in supplementary table S2.

The planned observation time was completed in $89.6 \%$ of patients at GAP stage I and $77.9 \%$ of patients at GAP stage II/III. In patients at GAP stage I, mean \pm SD duration of exposure to study medication was $10.5 \pm 3.3$ months and $11.3 \pm 2.1$ months in the nintedanib and placebo groups, respectively. In patients at

TABLE 1 Baseline characteristics stratified by baseline GAP (gender, age, physiology) stage

\begin{tabular}{|c|c|c|c|c|}
\hline & \multicolumn{2}{|c|}{ GAP stage I } & \multicolumn{2}{|c|}{ GAP stage II/III } \\
\hline & Nintedanib & Placebo & Nintedanib & Placebo \\
\hline Subjects & 304 & 196 & 334 & 226 \\
\hline Age years & $62.9 \pm 8.1$ & $63.1 \pm 8.3$ & $70.0 \pm 6.6$ & $70.3 \pm 5.8$ \\
\hline Male & $208(68.4)$ & 132 (67.3) & 299 (89.5) & $202(89.4)$ \\
\hline \multicolumn{5}{|l|}{ Race } \\
\hline White & 173 (56.9) & $110(56.1)$ & $187(56.0)$ & $137(60.6)$ \\
\hline Asian & $94(30.9)$ & $60(30.6)$ & $100(29.9)$ & $68(30.1)$ \\
\hline Black & $0(0.0)$ & $0(0.0)$ & $2(0.6)$ & $0(0.0)$ \\
\hline Missing $\#$ & $37(12.2)$ & $26(13.3)$ & $45(13.5)$ & $21(9.3)$ \\
\hline Former or current smoker & $209(68.8)$ & 130 (66.3) & $255(76.3)$ & $171(75.7)$ \\
\hline FVC mL & $2883 \pm 823$ & $2957 \pm 911$ & $2559 \pm 656$ & $2537 \pm 645$ \\
\hline FVC \% predicted & $85.8 \pm 17.3$ & $87.4 \pm 18.9$ & $74.2 \pm 16.0$ & $72.3 \pm 14.2$ \\
\hline FEV1/FVC ratio & $81.4 \pm 5.6$ & $81.1 \pm 6.1$ & $81.9 \pm 6.1$ & $82.2 \pm 5.8$ \\
\hline DLco \% predicted & $54.0 \pm 13.8$ & $53.5 \pm 14.1$ & $41.4 \pm 10.0$ & $41.3 \pm 9.7$ \\
\hline SGRQ total score & $36.2 \pm 19.7$ & $38.2 \pm 19.3$ & $42.6 \pm 18.2$ & $40.7 \pm 17.8$ \\
\hline \multicolumn{5}{|c|}{$\begin{array}{l}\text { Data are presented as } n \text {, mean } \pm \text { SD or } n(\%) \text {. FVC: forced vital capacity; FEV } 1 \text { : forced expiratory volume in } \\
1 \mathrm{~s} ; \text { DLCO: diffusing capacity of the lung for carbon monoxide; SGRQ: St George's Respiratory } \\
\text { Questionnaire. \#: in France, regulations did not permit the collection of data on race; ๆ: } n=299 \text { for } \\
\text { nintedanib and } n=194 \text { for placebo in GAP stage I subgroup; } n=325 \text { for nintedanib and } n=224 \text { for placebo in } \\
\text { GAP stage II/III subgroup. }\end{array}$} \\
\hline
\end{tabular}


GAP stage II/III, mean \pm SD duration of exposure to study medication was $10.1 \pm 3.4$ months and $10.4 \pm 3.3$ months in the nintedanib and placebo groups, respectively.

\section{FVC}

In nintedanib-treated patients, the annual rate of decline in FVC was similar in patients at GAP stage I and GAP stage II/III at baseline $\left(-110.1 \mathrm{~mL} \cdot \mathrm{year}^{-1}\right.$ and $-116.6 \mathrm{~mL} \cdot \mathrm{year}^{-1}$, respectively) and in both subgroups was lower than in placebo-treated patients $\left(-218.5 \mathrm{~mL} \cdot \mathrm{year}^{-1}\right.$ and $-227.6 \mathrm{~mL} \cdot \mathrm{year}^{-1}$, respectively) (figure 1). The observed changes in FVC over time in each subgroup are shown in figure 2. The treatment effect of nintedanib on reducing the annual rate of decline in FVC was consistent between patients at GAP stage I and GAP stage II/III at baseline (treatment-by-time-by-subgroup interaction $\mathrm{p}=0.92$ ). The benefit of nintedanib on FVC in these GAP stage subgroups was consistent with the treatment effect in the overall population $\left(109.9 \mathrm{~mL} \cdot \mathrm{year}^{-1}\right.$, 95\% CI 75.9-144.0 mL·year ${ }^{-1}$; $\left.\mathrm{p}<0.0001\right)$ [8].

In the analysis of the annual rate of decline in FVC in patients at GAP stage I, II and III at baseline, the effect of nintedanib was numerically greater in patients at GAP stage III (treatment-by-time-by-subgroup interaction $\mathrm{p}=0.10$ ) (supplementary figure $\mathrm{S} 1$ ).

\section{Disease progression and mortality}

The treatment effect of nintedanib on disease progression, measured by time to absolute FVC decline $\geqslant 5 \%$ pred or death, or absolute FVC decline $\geqslant 10 \%$ pred or death, over 52 weeks, was consistent across subgroups of patients at GAP stage I and II/III at baseline (table 2) and across subgroups of patients at GAP stages I, II and III (supplementary table S3).

Based on the number of deaths observed versus the number of deaths predicted based on GAP stage, nintedanib was associated with a relative reduction in the risk of mortality of $26.7 \%$ compared with placebo (table 3 ).

\section{Acute exacerbation}

The proportion of patients who had an acute exacerbation was higher in patients at GAP stage II/III than at GAP stage I (8.4\% versus 3.0\%). In patients at GAP stage I, the proportion of patients with one or more acute exacerbations was $1.6 \%$ in the nintedanib group and $5.1 \%$ in the placebo group. In patients at GAP stage II/III, the proportion of patients with one or more acute exacerbations was $7.8 \%$ in the nintedanib group and $9.3 \%$ in the placebo group. In the overall pooled population, there was a numerical reduction in the risk of having a first acute exacerbation with nintedanib versus placebo (hazard ratio (HR) $0.64,95 \%$ CI $0.39-1.05 ; \mathrm{p}=0.08$ ) [8]. Nintedanib had a numerically greater effect on time to first acute exacerbation in patients at GAP stage I (HR 0.32, 95\% CI 0.11-0.93) than GAP stage II/III (HR 0.82, 95\% CI $0.46-1.47$ ); however, the treatment-by-subgroup interaction was not significant ( $\mathrm{p}=0.14$; figure 3 ). The effect of nintedanib on time to first acute exacerbation across subgroups of patients at GAP stage I, II and III is presented in supplementary table S4.

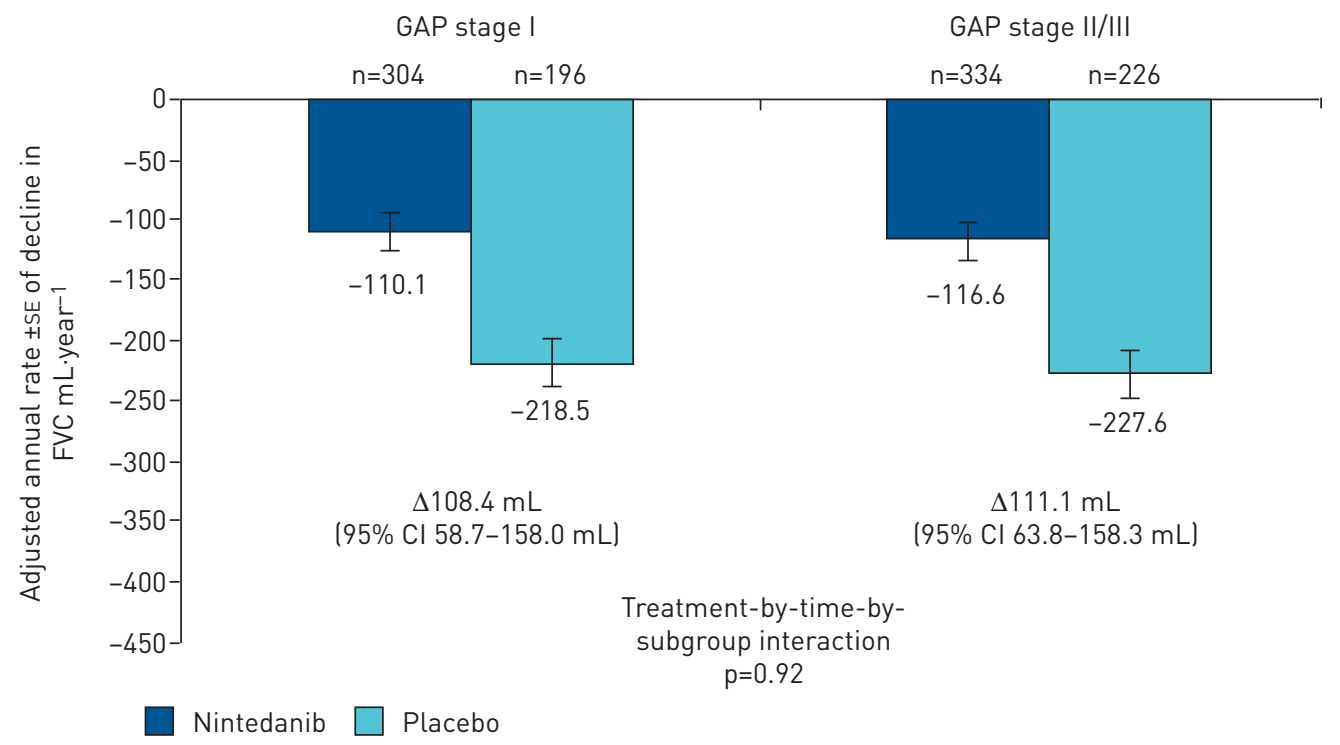

FIGURE 1 Annual rate of decline in forced vital capacity (FVC) by baseline GAP (gender, age, physiology) stage. 


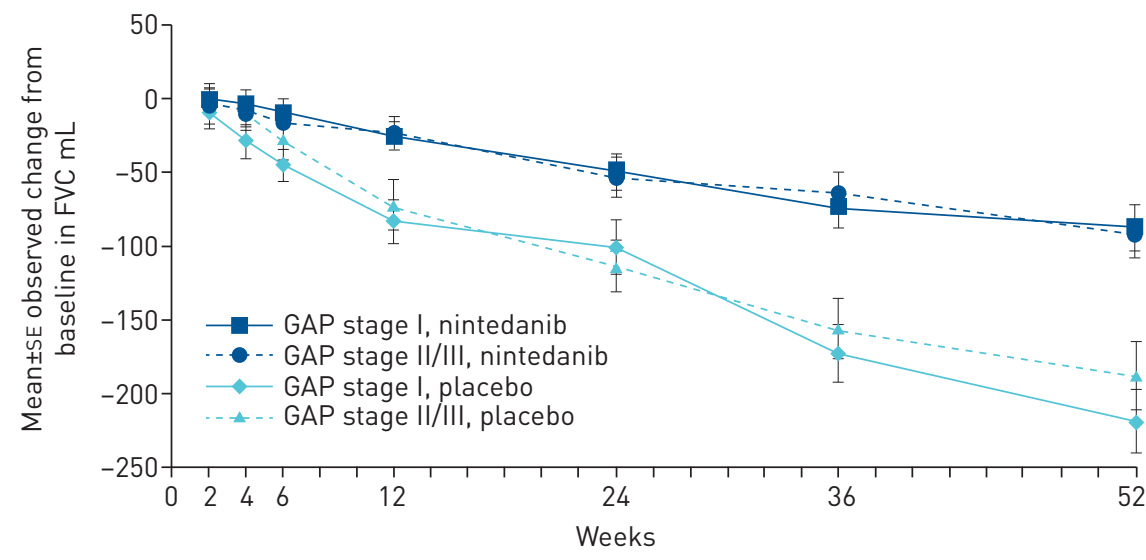

Patients $n$

GAP stage I, nintedanib

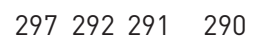

282

280

305

GAP stage II/III, nintedanib

GAP stage I, placebo

$329324322 \quad 314$

$196192193 \quad 192$

$220215213 \quad 210$

189

206

289

184

198

259

260

172

GAP stage II/III, placebo

FIGURE 2 Mean observed change from baseline in forced vital capacity (FVC) by baseline GAP (gender, age, physiologyl stage.

\section{Health-related quality of life}

Patients had worse SGRQ total scores at baseline with increasing GAP stage (37.0, 40.5 and 50.8 for patients at GAP stage I, II and III, respectively). There was no significant difference between the nintedanib and placebo groups in change from baseline in SGRQ total score at week 52 in the overall population $(-1.43,95 \% \mathrm{CI}-3.09-0.23$; $\mathrm{p}=0.09)$ [8].

The treatment effect of nintedanib on change from baseline in SGRQ total score seemed to be more pronounced in patients at GAP stage II/III versus patients at GAP stage I at baseline. The difference in adjusted mean change from baseline in SGRQ total score at week 52 between nintedanib and placebo was -2.67 (95\% CI $-5.07-0.27)$ and 0.09 (95\% CI -2.20-2.38) for patients at GAP stage II/III and GAP stage I, respectively (treatment-by-subgroup interaction $\mathrm{p}=0.06$; figure 4 ). Similarly, there was a more pronounced treatment effect in the activity and impact domains of the SGRQ across patients at GAP stages I and II/III ( $p=0.09$ and $p=0.12$, respectively), but not for the symptoms domain $(p=0.90)$ (supplementary table S5). Across patients at GAP stage I, II and III at baseline, the treatment effect was numerically greater with increasing GAP stage; however, the treatment-by-subgroup interaction was not significant ( $\mathrm{p}=0.21$; supplementary figure $\mathrm{S} 2$ ).

TABLE 2 Proportions of patients with disease progression over 52 weeks and hazard ratio for time to first event by baseline GAP (gender, age, physiology) stage

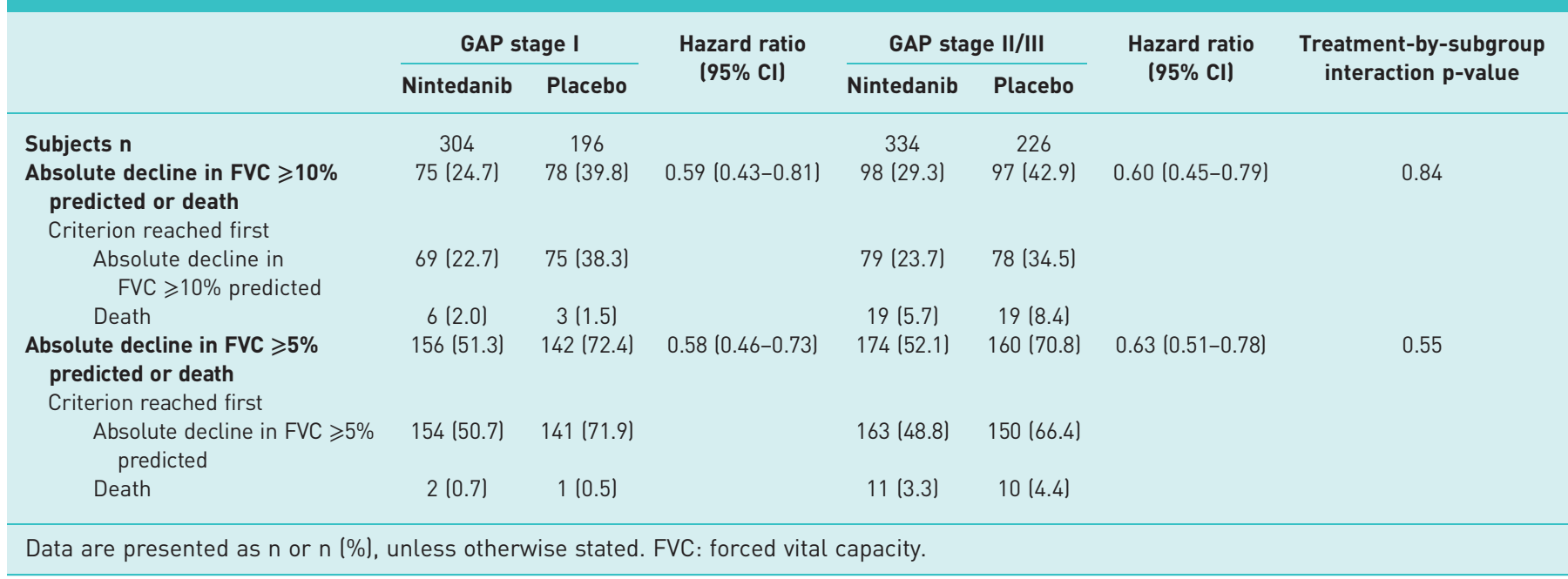




\begin{tabular}{|c|c|c|c|c|c|}
\hline & \multicolumn{4}{|c|}{ GAP stage } & \multirow{2}{*}{$\begin{array}{l}\text { Observed deaths as a proportion of } \\
\text { predicted deaths } \%\end{array}$} \\
\hline & I & II & III & Total & \\
\hline Nintedanib subjects & 304 & 296 & 38 & 638 & 43.8 \\
\hline Predicted deaths & 17.0 & 48.0 & 14.9 & 79.9 & \\
\hline Observed deaths & 9 & 23 & 3 & 35 & \\
\hline Placebo subjects & 196 & 193 & 33 & 422 & 59.8 \\
\hline Predicted deaths & 11.0 & 31.3 & 12.9 & 55.2 & \\
\hline Observed deaths & 7 & 19 & 7 & 33 & \\
\hline
\end{tabular}

\section{Safety and tolerability}

Data on adverse events are summarised in table 4. In patients at GAP stage I, patients who were treated with nintedanib were more likely to discontinue study drug prematurely than patients treated with placebo, with similar rates of discontinuation in nintedanib- and placebo-treated patients in the GAP stage II/III subgroups. Diarrhoea was the most frequent adverse event that resulted in permanent treatment discontinuation in patients treated with nintedanib. Serious and fatal adverse events within each subgroup by GAP stage are summarised in supplementary table S6.

\section{Discussion}

This post hoc subgroup analysis of pooled data from the INPULSIS ${ }^{\circledR}$ trials suggested that nintedanib had a similar benefit on the rate of FVC decline across patient subgroups stratified by baseline GAP stage I

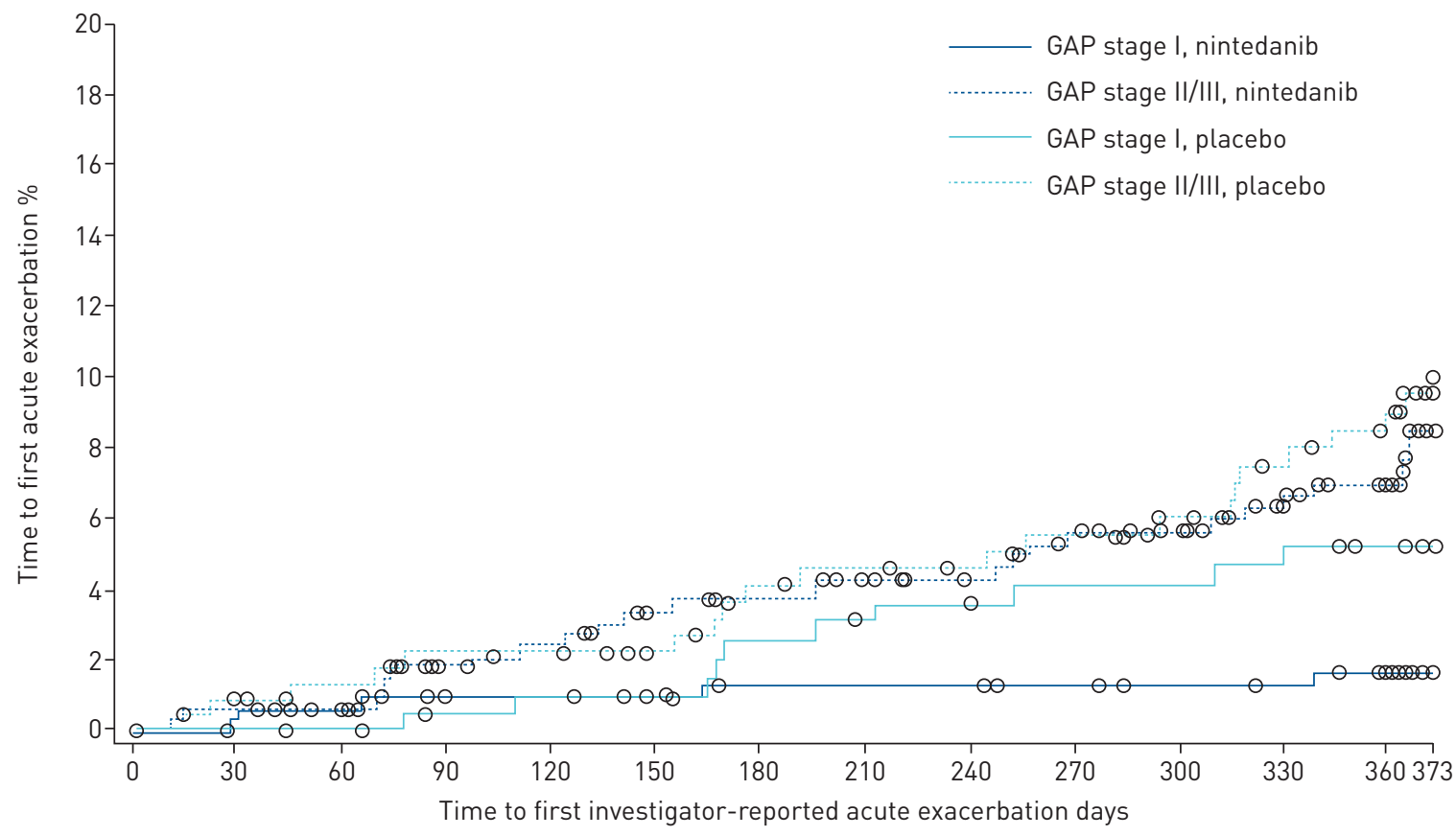

At risk $n$

GAP stage I, nintedanib 304

GAP stage II/III, nintedanib

GAP stage I, placebo

304
334
196
226

301
331
196
222

298
329
195
219

291
318
192
215

291
314
191
215

288
307
191
211

285
304
187
205

285
299
185
203

285
295
184
201

282
288
182
198

280
282
182
193

$\begin{array}{lll}279 & 273 & 254 \\ 274 & 264 & 238 \\ 181 & 178 & 174 \\ 186 & 181 & 167\end{array}$

FIGURE 3 Kaplan-Meier estimate of time to first investigator-reported acute exacerbation by baseline GAP (gender, age, physiologyl stage. 


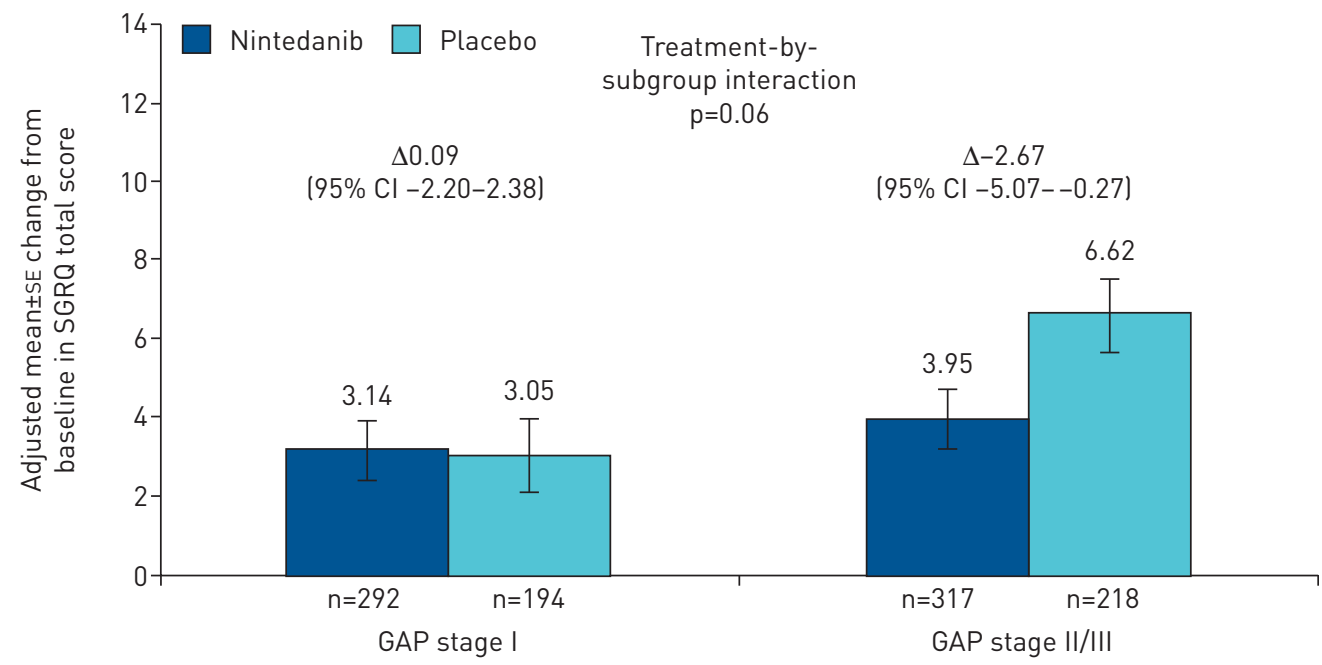

FIGURE 4 Change from baseline in St George's Respiratory Questionnaire (SGRQ) total score by subgroup at week 52 by baseline GAP (gender, age, physiology) stage.

TABLE 4 Tolerability and safety of nintedanib in subgroups by baseline GAP I gender, age, physiologyl stage

GAP stage I

\begin{tabular}{|c|c|c|c|c|}
\hline & \multicolumn{2}{|c|}{ GAP stage I } & \multicolumn{2}{|c|}{ GAP stage II/III } \\
\hline & Nintedanib & Placebo & Nintedanib & Placebo \\
\hline Subjects $\mathbf{n}$ & 304 & 196 & 334 & 226 \\
\hline Adverse event(s) & $289(95.1)$ & $177(90.3)$ & $320(95.8)$ & 201 (88.9) \\
\hline \multicolumn{5}{|l|}{ Most frequent adverse event(s)" } \\
\hline Diarrhoea & $188(61.8)$ & 34 (17.3) & $210(62.9)$ & 44 (19.5) \\
\hline Nausea & $79(26.0)$ & $13(6.6)$ & $77(23.1)$ & $15(6.6)$ \\
\hline Nasopharyngitis & $46(15.1)$ & $27(13.8)$ & 41 (12.3) & $41(18.1)$ \\
\hline Cough & $43(14.1)$ & 26 (13.3) & $42(12.6)$ & $31(13.7)$ \\
\hline Vomiting & $42(13.8)$ & $5(2.6)$ & $32(9.6)$ & $6(2.7)$ \\
\hline Decreased appetite & $23(7.6)$ & $7(3.6)$ & 45 (13.5) & $17(7.5)$ \\
\hline Progression of IPF" & $17(5.6)$ & $18(9.2)$ & $47(14.1)$ & $42(18.6)$ \\
\hline Weight decreased & $26(8.6)$ & $6(3.1)$ & $36(10.8)$ & $9(4.0)$ \\
\hline Upper respiratory tract infection & $23(7.6)$ & $21(10.7)$ & 35 (10.5) & $21(9.3)$ \\
\hline Abdominal pain & $32(10.5)$ & $5(2.6)$ & $24(7.2)$ & $5(2.2)$ \\
\hline Dyspnoea & $18(5.9)$ & $23(11.7)$ & $31(9.3)$ & $25(11.1)$ \\
\hline Bronchitis & $26(8.6)$ & $24(12.2)$ & $41(12.3)$ & $21(9.3)$ \\
\hline Serious adverse event(s) ${ }^{+}$ & 60 (19.7) & $45(23.0)$ & $134(40.1)$ & $81(35.8)$ \\
\hline Fatal adverse event(s) & $8(2.6)$ & $6(3.1)$ & $29(8.7)$ & $25(11.1)$ \\
\hline Adverse event(s) leading to treatment discontinuation & $48(15.8)$ & $16(8.2)$ & $75(22.5)$ & $39(17.3)$ \\
\hline \multicolumn{5}{|l|}{$\begin{array}{l}\text { Most frequent adverse event(s) leading to treatment } \\
\text { discontinuation }\end{array}$} \\
\hline Diarrhoea & 10 (3.3) & $1(0.5)$ & $18(5.4)$ & $0(0.0)$ \\
\hline Progression of IPF⿱ & $6(3.1)$ & $0(0.0)$ & $13(3.9)$ & $15(6.6)$ \\
\hline Nausea & 7 (2.3) & $0(0.0)$ & $6(1.8)$ & $0(0.0)$ \\
\hline Decreased appetite & $2(0.7)$ & $1(0.5)$ & $7(2.1)$ & $0(0.0)$ \\
\hline
\end{tabular}

Data are presented as $\mathrm{n}$ or $\mathrm{n}(\%)$ of patients treated with at least one dose of study drug with one or more such adverse event. IPF: idiopathic pulmonary fibrosis. " : adverse events reported in $>10 \%$ of patients in any subgroup; ": corresponds to MedDRA (Medical Dictionary for Regulatory Activities) term "IPF", which included disease worsening and acute exacerbations of IPF; ${ }^{+}$: an event that resulted in death, was immediately life-threatening, resulted in persistent or clinically significant disability or incapacity, required or prolonged hospitalisation, was related to a congenital anomaly or birth defect or was deemed serious for any other reason; ${ }^{\S}$ : adverse events leading to treatment discontinuation in $>2 \%$ of patients in any subgroup. 
versus II/III. These findings extend the results of previous studies that identified consistent benefits of nintedanib across many subgroups of patients with IPF [10-14, 21].

The GAP stage was developed as a mortality risk prediction tool in IPF, and has been suggested as a potential mechanism of cohort enrichment for mortality-focused clinical trials [7]. In the INPULSIS ${ }^{\circ}$ trials, fewer deaths occurred in both treatment groups than were predicted based on GAP stage at baseline, suggesting that the patients enrolled in these trials had lower 1-year mortality than the general population of patients with IPF. Based on the difference between observed and predicted deaths, nintedanib was associated with a relative reduction in the risk of 1 -year mortality of $26.7 \%$ versus placebo. Nintedanib resulted in a similar reduction versus placebo in the risk of disease progression (defined as an absolute FVC decline $\geqslant 10 \%$ pred or death) across GAP stages. There was a numerically greater benefit of nintedanib on disease progression in patients at GAP stage III than GAP stage I or II; however, the small number of patients at GAP stage III limited the robustness of this analysis. Thus our results suggest that GAP stage is unlikely to be a helpful mechanism for cohort enrichment for studies that are intended to show physiological benefit of antifibrotic medications with similar effects to nintedanib. This finding is consistent with recent data, suggesting that GAP stage does not predict physiologic disease progression in IPF [22]. Conversely, acute exacerbations of IPF were more common in patients at GAP stage II/III than at GAP stage I, consistent with previous studies reporting an increased risk of acute exacerbations in patients with more advanced disease [10, 23, 24], suggesting that GAP stage may be helpful to enrich cohorts for patients at higher risk of this outcome.

Pooled data from the INPULSIS ${ }^{\circledast}$ trials suggested that compared with placebo, nintedanib was associated with a numerical benefit on health-related quality of life measured by SGRQ score and in the risk of having a first investigator-reported acute exacerbation [8]. The current post hoc analysis suggests a greater treatment effect of nintedanib on quality of life in patients at GAP stage II/III compared with GAP stage I. The reasons for the potential difference across GAP stages are unclear. One possibility is that a similar rate of decline in FVC may have greater impact on quality of life in patients with more advanced disease, and thus the consistent effect of nintedanib on decline in FVC across GAP stages may have greater impact on quality of life in patients with more advanced disease. Similarly, there was a trend toward benefit of nintedanib on acute exacerbations of IPF in patients at GAP stage II/III compared with patients at GAP stage I. Additional studies are needed to evaluate whether nintedanib can improve quality of life or prevent acute exacerbations in specific patient subgroups.

The overall proportion of patients who had at least one adverse event was similar in patients at GAP stage I and GAP stage II/III and in the nintedanib and placebo arms within each subgroup. However, in patients at GAP stage I, the proportion of patients who prematurely discontinued study drug due to adverse events was nearly two-fold higher with nintedanib than with placebo, while premature discontinuations were similar with nintedanib and placebo in patients at GAP stage II/III. These findings suggest that patients with mild disease may have been less willing to accept adverse events than patients with more advanced disease. There are several potential explanations for this finding, including patient differences across GAP stages that impact tolerability of adverse events, greater acceptance of adverse events in patients with more advanced disease, and greater symptomatic benefit of preserving a given amount of lung function in patients with more advanced disease. A higher medication discontinuation rate in patients at GAP stage I may have implications for the design of clinical trials.

In summary, this post hoc analysis suggested that nintedanib has similar benefit on the rate of FVC decline between patients at GAP stage I versus II/III, indicating that GAP stage is unlikely to be a helpful tool for cohort enrichment in similarly designed clinical trials of pharmacotherapies for IPF. There were GAP stage-specific differences of unknown relevance in quality of life, time to first acute exacerbation and rate of discontinuation of nintedanib. This cohort included relatively few patients at GAP stage III and additional studies would be required to validate these findings and extend these results to a broader cohort.

Acknowledgements: Medical writing assistance, supported financially by Boehringer Ingelheim, was provided by Julie Fleming and Wendy Morris (FleishmanHillard Fishburn, London, UK), during the preparation of this article. The authors were fully responsible for all content and editorial decisions, were involved at all stages of manuscript development, and have approved the final version.

Conflict of interest: C.J. Ryerson reports grants and personal fees from Boehringer Ingelheim and Hoffmann-La Roche, outside the submitted work. M. Kolb reports research funding from the Canadian Pulmonary Fibrosis Foundation and the Canadian Institute for Health Research, acting as a site principal investigator in industry-sponsored clinical trials for Roche, Sanofi and Boehringer Ingelheim, grants from and service on an advisory board for the Pulmonary Fibrosis Foundation, advisory board fees from Boehringer Ingelheim, GlaxoSmithKline, AstraZeneca, Vertex, Genoa, Gilead and Alkermes, and grants and advisory board fees from Roche Canada and Janssen, personal fees from Prometic, outside the submitted work. L. Richeldi reports grants and personal fees from Boehringer Ingelheim during the conduct of the 
study; and grants and personal fees from InterMune, and personal fees from Medimmune, Biogen-Idec, Sanofi-Aventis Roche, Takeda, ImmuneWorks and Shionogi, outside the submitted work. J. Lee reports grants from the NIH, and personal fees from Genentech and Celgene, outside the submitted work. D. Wachtlin is an employee of Boehringer Ingelheim S. Stowasser is an employee of Boehringer Ingelheim. V. Poletti reports personal fees from Boehringer Ingelheim during the conduct of the study.

Support statement: The INPULSIS trials were funded by Boehringer Ingelheim. Funding information for this article has been deposited in the Crossref Funder Registry.

\section{References}

1 Raghu G, Remy-Jardin M, Myers JL, et al. Diagnosis of idiopathic pulmonary fibrosis. An official ATS/ERS/JRS/ ALAT clinical practice guideline. Am J Respir Crit Care Med 2018; 198: e44-e68.

2 Ley B, Collard HR, King TE Jr. Clinical course and prediction of survival in idiopathic pulmonary fibrosis. Am J Respir Crit Care Med 2011; 183: 431-440.

3 Zappala CJ, Latsi PI, Nicholson AG, et al. Marginal decline in forced vital capacity is associated with a poor outcome in idiopathic pulmonary fibrosis. Eur Respir J 2010; 35: 830-836.

4 du Bois RM, Weycker D, Albera C, et al. Forced vital capacity in patients with idiopathic pulmonary fibrosis: test properties and minimal clinically important difference. Am J Respir Crit Care Med 2011; 184: 1382-1389.

5 Sharp C, Adamali HI, Millar AB. A comparison of published multidimensional indices to predict outcome in idiopathic pulmonary fibrosis. ERJ Open Res 2017; 3: 00096-2016.

6 Paterniti MO, Bi Y, Rekić D, et al. Acute exacerbation and decline in forced vital capacity are associated with increased mortality in idiopathic pulmonary fibrosis. Ann Am Thorac Soc 2017; 14: 1395-1402.

7 Ley B, Ryerson CJ, Vittinghoff $\mathrm{E}$, et al. A multidimensional index and staging system for idiopathic pulmonary fibrosis. Ann Intern Med 2012; 156: 684-691.

8 Richeldi L, du Bois RM, Raghu G, et al. Efficacy and safety of nintedanib in idiopathic pulmonary fibrosis. $N$ Engl J Med 2014; 370: 2071-2082.

9 Richeldi L, Cottin V, du Bois RM, et al. Nintedanib in patients with idiopathic pulmonary fibrosis: combined evidence from the TOMORROW and INPULSIS ${ }^{\circ}$ trials. Respir Med 2016; 113: 74-79.

10 Costabel $\mathrm{U}$, Inoue $\mathrm{Y}$, Richeldi $\mathrm{L}$, et al. Efficacy of nintedanib in idiopathic pulmonary fibrosis across prespecified subgroups in INPULSIS. Am J Respir Crit Care Med 2016; 193: 178-185.

11 Kolb M, Richeldi L, Behr J, et al. Nintedanib in patients with idiopathic pulmonary fibrosis and preserved lung volume. Thorax 2017; 72: 340-346.

12 Raghu G, Wells AU, Nicholson AG, et al. Effect of nintedanib in subgroups of idiopathic pulmonary fibrosis by diagnostic criteria. Am J Respir Crit Care Med 2017; 195: 78-85.

13 Wuyts WA, Kolb M, Stowasser S, et al. First data on efficacy and safety of nintedanib in patients with idiopathic pulmonary fibrosis and forced vital capacity of $\leqslant 50 \%$ of predicted value. Lung 2016; 194: 739-743.

14 Taniguchi $\mathrm{H}, \mathrm{Xu} \mathrm{Z}$, Azuma A, et al. Subgroup analysis of Asian patients in the INPULSIS ${ }^{\oplus}$ trials of nintedanib in idiopathic pulmonary fibrosis. Respirology 2016; 21: 1425-1430.

15 Richeldi L, Cottin V, Flaherty KR, et al. Design of the INPULSIS ${ }^{\mathrm{TM}}$ trials: two phase 3 trials of nintedanib in patients with idiopathic pulmonary fibrosis. Respir Med 2014; 108: 1023-1030.

16 Miller MR, Hankinson J, Brusasco V, et al. Standardisation of spirometry. Eur Respir J 2005; 26: 319-338.

17 Macintyre N, Crapo RO, Viegi G, et al. Standardisation of the single-breath determination of carbon monoxide uptake in the lung. Eur Respir J 2005; 26: 720-735.

18 Jones PW, Quirk FH, Baveystock CM. The St George's Respiratory Questionnaire. Respir Med 1991; 85: Suppl. B, 25-31.

19 Swigris JJ, Brown KK, Behr J, et al. The SF-36 and SGRQ: validity and first look at minimum important differences in IPF. Respir Med 2010; 104: 296-304.

20 Swigris JJ, Wilson H, Esser D, et al. Psychometric properties of the St George's Respiratory Questionnaire in patients with idiopathic pulmonary fibrosis: insights from the INPULSIS trials. BMJ Open Respir Res 2018; 5 : e00278.

21 Wells A, Behr J, Stansen W, et al. Effect of baseline composite physiologic index on benefit of nintedanib in IPF Eur Respir J 2016; 48: Suppl. 60, OA1811.

22 Ley B, Bradford WZ, Vittinghoff E, et al. Predictors of mortality poorly predict common measures of disease progression in idiopathic pulmonary fibrosis. Am J Respir Crit Care Med 2016; 194: 711-718.

23 Collard HR, Ryerson CJ, Corte TJ, et al. Acute exacerbation of idiopathic pulmonary fibrosis. An International Working Group Report. Am J Respir Crit Care Med 2016; 194: 265-275.

24 Collard HR, Richeldi L, Kim DS, et al. Acute exacerbations in the INPULSIS trials of nintedanib in idiopathic pulmonary fibrosis. Eur Respir J 2017; 49: 1601339. 